\title{
TEMPERED BOEHMIANS AND ULTRADISTRIBUTIONS
}

\author{
PIOTR MIKUSIŃSKI
}

(Communicated by J. Marshall Ash)

\begin{abstract}
An extension of the Fourier transform which is a one-to-one continuous mapping from the space of tempered Boehmians onto the space of Schwartz distributions is introduced. This shows that the space of tempered Boehmians can be identified with the space $\mathscr{Z}^{\prime}$ of ultradistributions.
\end{abstract}

\section{INTRODUCTION}

The name Boehmians is used for all objects obtained by an abstract algebraic construction similar to the construction of the field of quotients [3]. The main difference is that it can be applied even if the "multiplication" has divisors of zero. Roughly speaking, instead of the usual quotients, one uses "quotients of sequences" $f_{n} / \phi_{n}$ where terms of the sequence in the numerator belong to some set $\mathscr{G}$ and the denominator is a "delta sequence", and we have $f_{n} * \phi_{m}=f_{m} * \phi_{n}$ for all $m, n \in \mathbb{N}$. Application of this construction to function spaces with. the convolution product yields various spaces of generalized functions. The obtained spaces contain the standard spaces of generalized functions defined as dual spaces. For example, if $\mathscr{G}=\mathscr{C}\left(\mathbb{R}^{N}\right)$ and delta sequences are defined as sequences of functions $\phi_{n} \in \mathscr{D}$ such that

$$
\begin{aligned}
& \int \phi_{n}=1 \text { for all } n \in \mathbb{N}, \\
& \int\left|\phi_{n}\right| \leq C \text { for some constant } C \text { and all } n \in \mathbb{N}, \\
& \operatorname{supp} \phi_{n} \rightarrow 0 \text { as } n \rightarrow \infty,
\end{aligned}
$$

then the obtained space of Boehmians properly contains the space of Schwartz distributions $\mathscr{D}^{\prime}[4,5]$.

Since Boehmians are represented by convolution quotients, integral transforms have natural extensions onto appropriately defined spaces of Boehmians $[6,8,9,10]$. The space of tempered Boehmians was introduced in [7] as one for which the Fourier transform can be well defined. In this case $\mathscr{G}$ is the space of slowly increasing functions (functions bounded by polynomials) and delta sequences are defined as above. This space of Boehmians properly contains the space of tempered distributions $\mathscr{S}^{\prime}$. The Fourier transforms of tempered Boehmians form a proper subspace of the space of Schwartz distributions $\mathscr{D}^{\prime}$. In this note we modify slightly the definition of tempered Boehmians given

Received by the editors March 31, 1993 and, in revised form, June 14, 1993.

1991 Mathematics Subject Classification. Primary 44A40, 42B10; Secondary 46F05.

Key words and phrases. Boehmians, ultradistributions, Fourier transform. 
in [7] by changing the class of delta sequences. This time delta sequences are formed from rapidly decreasing functions and the third condition is replaced by

$$
\lim _{n \rightarrow \infty} \int_{\|x\| \geq \varepsilon}\|x\|^{k}\left|\phi_{n}(x)\right| d x=0 \quad \text { for every } k \in \mathbb{N} \text { and } \varepsilon>0 .
$$

The new space of tempered Boehmians is larger than the one defined in [7]. The Fourier transform can be extended to the new space, and all its usual properties are preserved. But this change produced also a rather surprising and desirable result: every Schwartz distribution is now the Fourier transform of a tempered Boehmian. Consequently, the space of tempered Boehmians can be identified with the space $\mathscr{Z}^{\prime}$ of ultradistributions defined by Ehrenpreis [1] and Gelfand and Shilov [2] (see also [11] for a nice exposition). This gives an interesting characterization of $\mathscr{Z}^{\prime}$ by convolution quotients. It is also the first example of a space of Boehmians which is identical with a space of generalized functions defined as a dual space.

Note that the space of tempered Boehmians is defined algebraically, without the necessity of introducing topology on the space of test functions. We thus have an algebraic characterization of $\mathscr{Z}^{\prime}$.

\section{TEMPERED BOEHMiANS}

A complex-valued infinitely differentiable function $f$ defined on $\mathbb{R}^{N}$ is called rapidly decreasing if

$$
\sup _{|\alpha| \leq m} \sup _{x \in \mathbb{R}^{N}}\left(1+x_{1}^{2}+\cdots+x_{N}^{2}\right)^{m}\left|D^{\alpha} f(x)\right|<\infty
$$

for every nonnegative integer $m$. Here $\alpha=\left(\alpha_{1}, \ldots, \alpha_{N}\right), \alpha_{N}$ are nonnegative integers, $|\alpha|=\left|\alpha_{1}\right|+\cdots+\left|\alpha_{N}\right|$, and

$$
D^{\alpha}=\frac{\partial^{|\alpha|}}{\partial x^{\alpha}}=\frac{\partial^{|\alpha|}}{\partial x_{1}^{\alpha_{1}} \cdots \partial x_{N}^{\alpha_{N}}} .
$$

The space of all rapidly decreasing functions on $\mathbb{R}^{N}$ is denoted by $\mathscr{S}$. By a delta sequence we mean a sequence of real-valued functions $\phi_{1}, \phi_{2}, \ldots \in \mathscr{S}$ such that

$\int \phi_{n}=1$ for all $n \in \mathbb{N}$,

$\int\left|\phi_{n}\right| \leq C$ for some constant $C$ and all $n \in \mathbb{N}$,

$\lim _{n \rightarrow \infty} \int_{\| x|| \geq \varepsilon}\|x\|^{k}\left|\phi_{n}(x)\right| d x=0$ for every $k \in \mathbb{N}$ and $\varepsilon>0$.

Note that if $\phi \in \mathscr{S}$ and $\int \phi=1$, then the sequence of functions $\phi_{n}(x)=$ $n^{N} \phi(n x)$ is a delta sequence.

The Fourier transform of $\phi \in \mathscr{S}$ is denoted by $\hat{\phi}$, i.e.,

$$
\hat{\phi}(s)=\int \phi(x) e^{-i(x \cdot s)} d x .
$$

We will use the fact that if $\left\{\phi_{n}\right\}$ is a delta sequence, then $\hat{\phi}_{n} \rightarrow 1$ uniformly on compact subsets of $\mathbb{R}^{N}$.

A complex-valued function $f$ on $\mathbb{R}^{N}$ is called slowly increasing if there exists a polynomial $p$ on $\mathbb{R}^{N}$ such that the function $f(x) / p(x)$ is bounded. The space of all slowly increasing continuous functions on $\mathbb{R}^{N}$ is denoted by $\mathscr{T}$. 
By the convolution $f * g$ of two functions $f$ and $g$ we mean the function

$$
(f * g)(x)=\int f(u) g(x-u) d u
$$

whenever the integral exists.

A pair of sequences $\left(f_{n}, \phi_{n}\right), n=1,2, \ldots$, is called a quotient of sequences and is denoted by $f_{n} / \phi_{n}$ if $f_{n} \in \mathscr{T},\left\{\phi_{n}\right\}$ is a delta sequence, and $f_{n} * \phi_{m}=$ $f_{m} * \phi_{n}$ for all $m, n \in \mathbb{N}$. Two quotients of sequences $f_{n} / \phi_{n}$ and $g_{n} / \psi_{n}$ are equivalent if $f_{n} * \psi_{n}=g_{n} * \phi_{n}$ for every $n \in \mathbb{N}$. The equivalence class of a quotient of sequences $f_{n} / \phi_{n}$ will be denoted by $\left[f_{n} / \phi_{n}\right]$. The space of equivalence classes of quotients of sequences will be denoted by $\mathfrak{B}_{\mathfrak{G}}$, and its elements will be called tempered Boehmians.

$\mathfrak{B}_{\mathscr{G}}$ is a vector space with addition and multiplication by a scalar defined as follows:

$\lambda\left[f_{n} / \phi_{n}\right]=\left[\lambda f_{n} / \phi_{n}\right]$ and $\left[f_{n} / \phi_{n}\right]+\left[g_{n} / \psi_{n}\right]=\left[\left(f_{n} * \psi_{n}+g_{n} * \phi_{n}\right) /\left(\phi_{n} * \psi_{n}\right)\right]$.

A function $f \in \mathscr{T}$ can be identified with the Boehmian $\left[\left(f * \phi_{n}\right) / \phi_{n}\right]$, where $\left\{\phi_{n}\right\}$ is any delta sequence. It can be shown that this identification is independent of the choice of the delta sequence $\left\{\phi_{n}\right\}$.

For $F=\left[f_{n} / \phi_{n}\right] \in \mathfrak{B}_{\mathscr{g}}$ we define $D^{\alpha} F=\left[\left(f_{n} * D^{\alpha} \phi_{n}\right) /\left(\phi_{n} * \phi_{n}\right)\right]$. Note that $D^{\alpha} F \in \mathfrak{B}_{g}$ and that the defined operation agrees with the usual differentiation if $F$ is a Boehmian corresponding to a differentiable function.

If $F=\left[f_{n} / \phi_{n}\right] \in \mathfrak{B}_{g}$ and $f_{n} \in \mathscr{S}$ for all $n \in \mathbb{N}$, then $F$ is called a rapidly decreasing Boehmian. The space of all rapidly decreasing Boehmians is denoted by $\mathfrak{B}_{\mathscr{S}}$. If $F=\left[f_{n} / \phi_{n}\right] \in \mathfrak{B}_{\mathscr{G}}$ and $G=\left[g_{n} / \gamma_{n}\right] \in \mathfrak{B}_{\mathscr{S}}$, then we can define the convolution $F * G$ as $F * G=\left[\left(f_{n} * g_{n}\right) /\left(\phi_{n} * \gamma_{n}\right)\right]$. Note that $F * G \in \mathfrak{B}_{\mathscr{g}}$.

\section{THE FOURIER TRANSFORM OF TEMPERED BOEHMIANS}

The Fourier transform of a tempered Boehmian is a Schwartz distribution. The space of test functions with compact support in $\mathbb{R}^{N}$ is denoted by $\mathscr{D}$, and the space of distributions is denoted by $\mathscr{D}^{\prime}$. The Fourier transform $\hat{f}$ of a slowly increasing function $f$ is the distribution defined by $\langle\hat{f}, \phi\rangle=\langle f, \hat{\phi}\rangle$.

The proofs of Theorems 3.1 and 3.3 can be found in [7]. The space of tempered Boehmians considered in this note is more general than the one in [7], but the proofs presented there can be used in this setting practically without any changes.

3.1. Theorem. If $\left[f_{n} / \phi_{n}\right] \in \mathfrak{B}_{\mathscr{g}}$, then the sequence $\left\{\hat{f}_{n}\right\}$ converges in $\mathscr{D}^{\prime}$. Moreover, if $\left[f_{n} / \phi_{n}\right]=\left[g_{n} / \gamma_{n}\right] \in \mathfrak{B}_{g}$, then the sequences $\left\{\hat{f}_{n}\right\}$ and $\left\{\hat{g}_{n}\right\}$ have the same limit.

3.2. Definition. By the Fourier transform $\hat{F}$ of a tempered Boehmian $F=$ $\left[f_{n} / \phi_{n}\right]$ we mean the limit of $\left\{\hat{f}_{n}\right\}$ in $\mathscr{D}^{\prime}$.

The defined Fourier transform is thus a linear mapping from $\mathfrak{B}_{\mathscr{g}}$ into $\mathscr{D}^{\prime}$.

3.3. Theorem. Let $F=\left[f_{n} / \phi_{n}\right] \in \mathfrak{B}_{\mathscr{g}}$ and $G=\left[g_{n} / \gamma_{n}\right] \in \mathfrak{B}_{\mathscr{S}}$. Then:

(a) $\left(\frac{\partial}{\partial x_{m} F}\right)^{\wedge}=i x_{m} \hat{F}$,

(b) $\hat{G}$ is an infinitely differentiable function,

(c) $(F * G)^{\wedge}=\hat{F} \hat{G}$, and

(d) $\hat{F} \hat{\phi}_{n}=\hat{f}_{n}$ for all $n \in \mathbb{N}$. 
3.4. Theorem. Every distribution is the Fourier transform of a tempered Boehmian.

Proof. Let $\psi \in \mathscr{D}$ and $\psi(0)=1$. Let $\phi=(2 \pi)^{-N} \hat{\psi}$ and $\phi_{n}(x)=n^{N} \phi(n x)$, $n \in \mathbb{N}$. Then $\left\{\phi_{n}\right\}$ is a delta sequence such that $\hat{\phi}_{n}$ have compact support. Let $f$ be an arbitrary distribution. Since $f \hat{\phi}_{n}$ have compact supports, there exist tempered distributions $g_{n}$ such that $\hat{g}_{n}=f \hat{\phi}_{n}$. Now define $F=$ $\left[\left(g_{n} * \phi_{n}\right) /\left(\phi_{n} * \phi_{n}\right)\right]$. Since $g_{n} * \phi_{n} \in \mathscr{T}, F \in \mathfrak{B}_{\mathscr{G}}$. Moreover,

$$
\hat{F}=\lim _{n \rightarrow \infty}\left(g_{n} * \phi_{n}\right)^{\wedge}=\lim _{n \rightarrow \infty} \hat{g}_{n} \hat{\phi}_{n}=\lim _{n \rightarrow \infty} f \hat{\phi}_{n} \hat{\phi}_{n}=f \text {. }
$$

The proof of the above theorem suggests an inversion formula for the extended Fourier transform:

$$
F=\left[\left(\left(\hat{F} \hat{\phi}_{n}\right)^{\vee} * \phi_{n}\right) /\left(\phi_{n} * \phi_{n}\right)\right],
$$

where $\left\{\phi_{n}\right\}$ is a delta sequence such that $\hat{F} \hat{\phi}_{n}$ are tempered distributions and $\left(\hat{F} \hat{\phi}_{n}\right)^{\vee}$ is the distributional inverse Fourier transform of $\hat{F} \hat{\phi}_{n}$.

Let $f \in \mathscr{D}^{\prime}$, and let $\psi_{n} \in \mathscr{D}$ be such that $\left\{\hat{\psi}_{n}\right\}$ is a delta sequence. Define

$$
\hat{f}=\left[\left(\left(f \psi_{n}\right)^{\wedge} * \hat{\psi}_{n}\right) /\left(\hat{\psi}_{n} * \hat{\psi}_{n}\right)\right] \text {. }
$$

It is easy to check that $\hat{f}$ is a tempered Boehmian. Consequently, the Fourier transform of an arbitrary distribution can be defined as a tempered Boehmian.

\section{CONTINUITY OF THE FOURIER TRANSFORM}

Now we are going to define a convergence in $\mathfrak{B}_{\mathscr{g}}$ and prove that the extended Fourier transform is continuous.

4.1. Definition. A sequence of functions $f_{n} \in \mathscr{T}$ is said to be convergent to $f$ in $\mathscr{T}$ if there exists a polynomial $p$ such that $\left(f_{n}-f\right) / p \rightarrow 0$ uniformly on $\mathbb{R}^{N}$.

Note that this convergence can be defined by a strict inductive limit of a sequence of Banach spaces:

$$
\begin{gathered}
E_{n}=\left\{f \in \mathscr{T}:|f(x)|\left(1+x_{1}^{2}+\cdots+x_{N}^{2}\right)^{-n} \text { is bounded }\right\}, \\
\|f\|_{n}=\sup _{x \in \mathbf{R}^{N}}|f(x)|\left(1+x_{1}^{2}+\cdots+x_{N}^{2}\right)^{-n} .
\end{gathered}
$$

4.2. Definition. A sequence of tempered Boehmians $\left\{F_{n}\right\}$ is said to be $\delta$ convergent to a tempered Boehmian $F$, denoted by $F_{n} \stackrel{\delta}{\rightarrow} F$, if there exists a delta sequence $\left\{\phi_{n}\right\}$ such that $F_{n} * \phi_{k}, F * \phi_{k} \in \mathscr{T}$, for every $k, n \in \mathbb{N}$, and $F_{n} * \phi_{k} \rightarrow F * \phi_{k}$ in $\mathscr{T}$ as $n \rightarrow \infty$.

In other words, a sequence of tempered Boehmians $F_{n}$ is $\delta$-convergent to $F$ if there exist a delta sequence $\left\{\phi_{n}\right\}$ and a polynomial $p$ such that, for every $k \in \mathbb{N},\left(\left(F_{n}-F\right) * \delta_{k}\right) / p \rightarrow 0$ uniformly on $\mathbb{R}^{N}$ as $n \rightarrow \infty$.

4.3. Theorem. If $F_{n} \stackrel{\delta}{\rightarrow} F$ in $\mathfrak{B}_{\mathcal{g}}$, then $\hat{F}_{n} \rightarrow \hat{F}$ in $\mathscr{D}^{\prime}$.

Proof. Since the Fourier transform is linear, we can assume that $F_{n} \stackrel{\delta}{\rightarrow} 0$. There exist a delta sequence $\left\{\delta_{n}\right\}$ and a polynomial $p$ such that, for every $k \in \mathbb{N}$, 
$\left(F_{n} * \delta_{k}\right) / p \rightarrow 0$ as $n \rightarrow \infty$ uniformly on $\mathbb{R}^{N}$. Let $\phi \in \mathscr{D}$. Then there exist a $k \in \mathbb{N}$ and a $\psi \in \mathscr{D}$ such that $\hat{\delta}_{k}(x) \psi(x)=1$ on the support of $\phi$. Then

$$
\begin{aligned}
\left\langle\hat{F}_{n}, \phi\right\rangle & =\left\langle\hat{F}_{n}, \hat{\delta}_{k} \psi \phi\right\rangle=\left\langle\hat{F}_{n} \hat{\delta}_{k}, \psi \phi\right\rangle=\left\langle F_{n} * \delta_{k},(\psi \phi)^{\wedge}\right\rangle \\
& =\left\langle\left(F_{n} * \delta_{k}\right) / p, p(\psi \phi)^{\wedge}\right\rangle \rightarrow 0 \quad \text { as } n \rightarrow \infty,
\end{aligned}
$$

because $\left(F_{n} * \delta_{k}\right) / p \rightarrow 0$ as $n \rightarrow \infty$ uniformly on $\mathbb{R}^{N}$ and $p(\psi \phi)^{\wedge}$ is a rapidly decreasing function.

\section{REFERENCES}

1. L. Ehrenpreis, Analytic functions and the Fourier transform of distributions. I, Ann. of Math. (2) 63 (1956), 129-159.

2. I. M. Gelfand and G. E. Shilov, Generalized functions, Vols. 1-3, Academic Press, New York, 1964.

3. J. Mikusiński and P. Mikusiński, Quotients de suites et leurs applications dans l'analyse fonctionnelle, C.R. Acad. Sci. Paris Sér. I Math. 293 (1981), 463-464.

4. P. Mikusiński, Convergence of Boehmians, Japan. J. Math. 9 (1983), 159-179.

5. __ Boehmians and generalized functions, Acta Math. Hungar. 51 (1988), 271-281.

6. __ Fourier transform for integrable Boehmians, Rocky Mountain J. Math. 17 (1987), 577-582.

7. _ The Fourier transform of tempered Boehmians, Fourier Analysis, Lecture Notes in Pure and Appl. Math., Marcel Dekker, New York, 1994, pp. 303-309.

8. P. Mikusiński, A. Morse, and D. Nemzer, The two-sided Laplace transform for Boehmians, submitted.

9. P. Mikusinski and A. Zayed, The Radon transform of Boehmians, Proc. Amer. Math. Soc. 118 (1993), 561-570.

10. D. Nemzer, The Laplace transform on a class of Boehmians, Bull. Austral. Math. Soc. 46 (1992), 347-352.

11. A. H. Zemanian, Distribution theory and transform analysis, Dover, New York, 1987.

Department of Mathematics, University of Central Florida, Orlando, Florida 32816-1364

E-mail address: piotrm@pegasus.cc.ucf.edu 